\title{
An Overview of Recent Deeply Virtual Compton Scattering Results at HERMES
}

\author{
David Francis Mahon \\ (on behalf of the HERMES Collaboration) \\ University of Glasgow, Glasgow, G12 8QQ, Scotland, UK
}

\begin{abstract}
We present recently-released HERMES Deeply Virtual Compton Scattering results of Beam-Helicity (formerly Beam-Spin, BSA) and Beam-Charge (BCA) asymmetries from unpolarised proton, deuteron and selected heavy nuclear gas targets. A new analysis technique utilising the ability of HERA to produce a longitudinally-polarised lepton beam of both charges has been used to simultaneously extract the BSA and BCA amplitudes arising from the DVCS-squared and Interference terms of the total cross section for proton and deuteron data. Results of the Transverse Target-Spin Asymmetry (TTSA) obtained using this technique have been published for transverselypolarised proton data. These extracted asymmetries are presented, integrated over the kinematic acceptance of the HERMES experiment for this interaction, with their dependences on $-t, x_{B}$ and $Q^{2}$ also shown. The results are compared with asymmetries from a phenomenological model of GPDs based on double distributions.
\end{abstract}

Keywords: DVCS, proton, deuteron

PACS: 13.60.-r 13.60.Fz 14.20.Dh 24.85.+p

\section{INTRODUCTION}

Hard exclusive electroproduction of real photons i.e. Deeply Virtual Compton Scattering (DVCS), is regarded as the simplest process in which to access information on Generalised Parton Distributions (GPDs) [1, 2], an informational framework encompassing Form Factors (FFs) and Parton Distribution Functions (PDFs) as moments and limiting cases respectively in order to construct a coherent view of the structure of the nucleon. Interest was sparked by Ji [3] who discovered that the unknown total angular momentum of quarks could be deduced by investigating GPDs:

$$
J_{q}=\frac{1}{2} \lim _{t \rightarrow 0} \int_{-1}^{1} x\left[H_{q}(x, \xi, t)+E_{q}(x, \xi, t)\right] d x
$$

Measurements at HERMES of the $e+P \rightarrow e+P+\gamma$ process have two contributions to the cross section. One is from DVCS and the other from Bethe-Heitler $(\mathrm{BH})$ scattering which does not provide information about GPDs. Although the BH contribution dominates, the interference, $I$ in the scattering amplitudes enhances the contribution from the DVCS process. By measuring the interference amplitudes HERMES can determine useful information about GPDs. The information is encoded as Fourier amplitudes in the azimuthal distribution of the real photons produced during the process. These amplitudes are functions of kinematic variables and Compton Form Factors (CFFs) which are GPDs convoluted with a hard scattering kernel. 


\section{AZIMUTHAL ASYMMETRIES AT HERMES}

\section{Combined Beam-Helicity and Beam-Charge Extraction}

Applying a new analytical technique described in Ref. [4] using data taken with both beam charges and spin orientations, the 'Combined' Beam Charge (BCA) and Beam-Helicity (formerly Beam-Spin Asymmetry, BSA) asymmetries were extracted from unpolarised proton and deuteron targets. These are related to CFFs in a Fourier expansion in $\phi$ arising from the squared-DVCS $\left(A_{L U}^{\mathrm{DVCS}}\right)$ and interference terms $\left(A_{L U}^{I}\right.$ and $A_{C}$ ). The asymmetries are extracted using the Maximum Likelihood method using the following expansions relating to the aforementioned Fourier amplitudes:

$$
\begin{gathered}
A_{C}(\phi) \equiv \sum_{n=0}^{3} A_{C}^{\cos (n \phi)} \cos (n \phi) \\
A_{L U}^{I}(\phi) \equiv \sum_{n=1}^{2} A_{L U, I}^{\sin (n \phi)} \sin (n \phi), \quad A_{L U}^{D V C S}(\phi) \equiv A_{L U, \mathrm{DVCS}}^{\sin (\phi)} \sin (\phi)
\end{gathered}
$$

This analysis for the first time allows the pure interference contribution to the BSA, $A_{L U}^{I}$ to be extracted, providing unique access to the linear combination of leading-order CFFs arising from the interference term. Refs. [5] and [6] outline the sensitivity, at HERMES kinematics of the leading sinusoidal, $s_{1}^{I}$ and cosinusoidal, $c_{1}^{I}$ Fourier amplitudes of the BSA and BCA to the imaginary and real parts respectively of the leading-twist CFF $\mathscr{H}$ as seen in Eqs. 4 and 5.

$$
\begin{aligned}
s_{1}^{I} & \propto \mathfrak{I} m\left\{F_{1} \mathscr{H}+\frac{x_{B}}{2-x_{B}}\left(F_{1}+F_{2}\right) \widetilde{\mathscr{H}}-\frac{t}{4 M^{2}} F_{2} \mathscr{E}\right\} \\
c_{1}^{I} & \propto \operatorname{Re}\left\{F_{1} \mathscr{H}+\frac{x_{B}}{2-x_{B}}\left(F_{1}+F_{2}\right) \widetilde{\mathscr{H}}-\frac{t}{4 M^{2}} F_{2} \mathscr{E}\right\}
\end{aligned}
$$

The higher $A_{L U}^{I}$ harmonics and $A_{L U}^{\mathrm{DVCS}}$ amplitudes relate to higher-twist CFFs and are expected to be suppressed.

\section{Target-Spin Asymmetries}

The Transverse Target Spin Asymmetry, a function of target-polarisation and angles $\phi$ and $\phi_{s}$, is sensitive to CFF $\mathscr{E}$ from the $A_{U T}^{\sin \left(\phi-\phi_{s}\right) \cos (\phi)}$ amplitude, where $\phi_{s}$ is the azimuthal angle between the scattering and target-polarisation planes. Results are presented in Ref. [4] and will not be shown here. Current analysis of the Longitudinal Target-Spin Asymmetry from polarised proton and deuteron target performed at HERMES will provide sensitivity to the twist-2 polarised GPD $\widetilde{H}$ from the imaginary part of its corresponding CFF via the leading $A_{U L}^{\sin (\phi)}$ amplitude. 


\section{RESULTS}

The results of the combined beam-helicity and beam-charge asymmetry amplitudes arising from the interference term shown in Eqs. 2 and 3 are presented in Figs. 1 and 2 as a function of $-t, x_{B}, Q^{2}$ and integrated over all kinematics in the HERMES acceptance. The error bars and bands highlight the statistical and systematics uncertainties respectively, where the latter includes contributions from acceptance, smearing, finite bin-width and misalignment of the detector with respect to the beam direction. The analysed sample contains an average contamination of $\sim 13 \%$ from the associated process where an excited nucleon resonance occupies the final state. The results have been compared (not shown here) to theoretical predictions based on a GPD model from [7] using either a 'factorised' or 'Regge-inspired' ansätz for the $t$-dependence.

Fig. 1 shows a large $A_{C}^{\cos (\phi)}$ amplitude, sensitive to GPD $H$ and proportional to $-A_{C}^{\cos (0 \phi)}$ as expected for both targets. The theoretical predictions using the Regge ansätz without the ' $D$ '-term provide good agreement with the experimental data. The higher $\cos (n \phi)$ amplitudes relating to higher-twist terms are found to be compatible with zero. The results from both the proton and deuteron data are consistent except at the high $-t$, 'incoherent-enriched' region where the difference could be explained by the contribution from incoherent scattering on the neutron inside the deuteron.

The comparison of results from the BSA presented in Fig. 2 shows a large $A_{L U, I}^{\sin (\phi)}$ amplitude, also sensitive to GPD $H$. The 'constant' term in the top panel was added as a check of the fitting method and is zero as expected. The twist- $3 \sin (2 \phi)$ term is suppressed with respect to the leading order amplitude. Currently no explanation can be given for the opposite sign of this amplitude for proton and deuteron data. The $A_{L U, \mathrm{DVCS}}^{\sin (\phi)}$ amplitude (not shown here) was found to be compatible with zero.

Recent results from DVCS off selected nuclear target gases [8] such as Neon, Krypton and Xenon also suggest there is no nuclear mass dependence of the BCA or BSA.

\section{ACKNOWLEDGMENTS}

I gratefully acknowledge the HERMES management for encouraging me to attend CIPANP '09 and the University of Glasgow and various funding agencies for their financial support.

\section{REFERENCES}

1. F. M. Dittes, et al. Phys. Lett., B209:325-329, 1988.

2. D. Mueller, et al. Fortschritte der Physik, 42:101, 1994.

3. Ji, Xiangdong. Phys. Rev. Lett., 1997: 610.

4. Airapetian, A. et al. JHEP, 06:066, 0802.2499 (2008)

5. A. V. Belitsky, D. Muller, and A. Kirchner. Nuclear Physics B, 629:323, 2002.

6. M. Diehl. hep-ph/0307382, DESY-THESIS-2003-018.

7. Vanderhaeghen, M., M. Guidal, and P.A.M. Guichon. Physical Review D, 1999: 094017. 1999, pp. 651-654.

8. Airapetian, A. et al. Unpublished Draft 


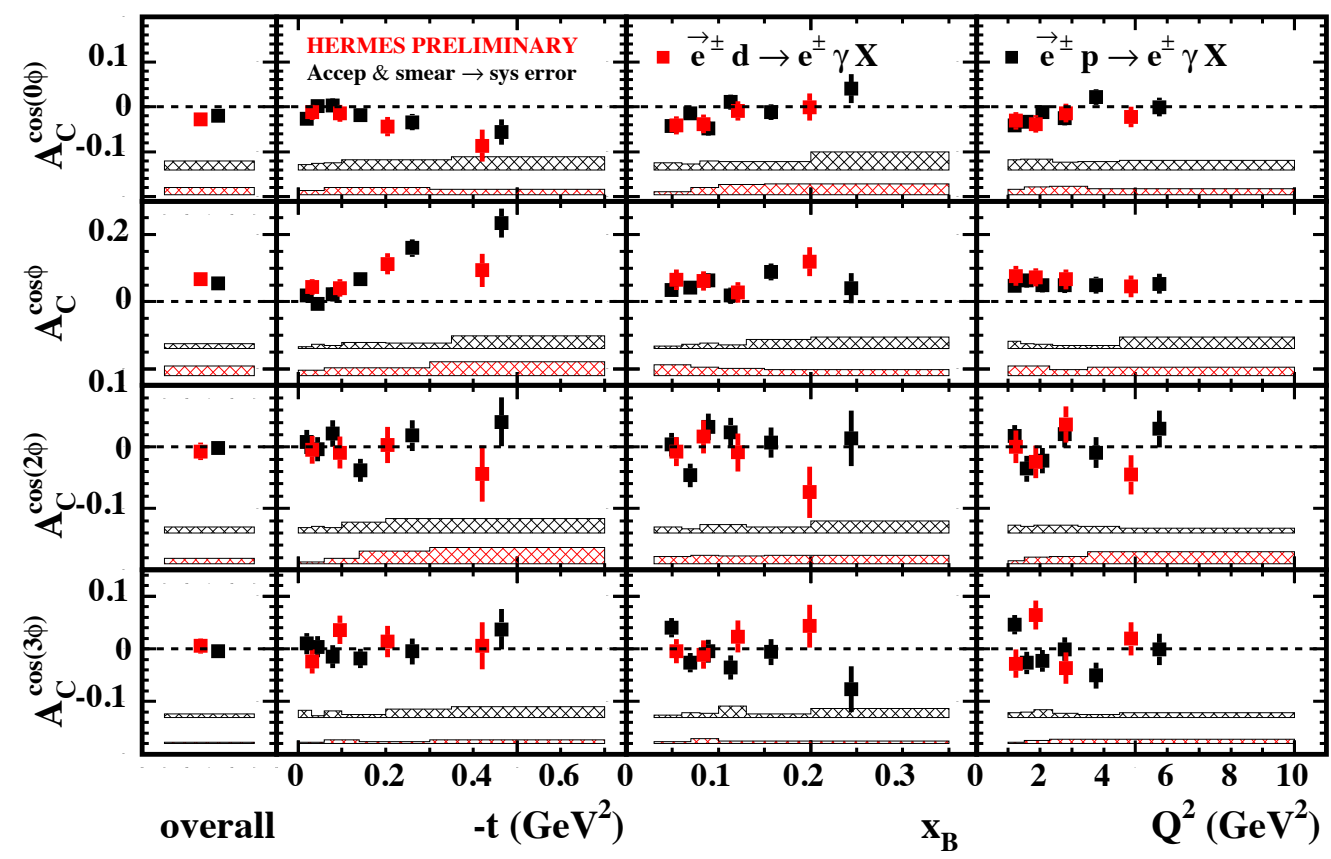

FIGURE 1. Comparison of the extracted BCA amplitudes from HERMES unpolarised proton and deuteron data as a function of $-t, x_{B}$ and $Q^{2}$.

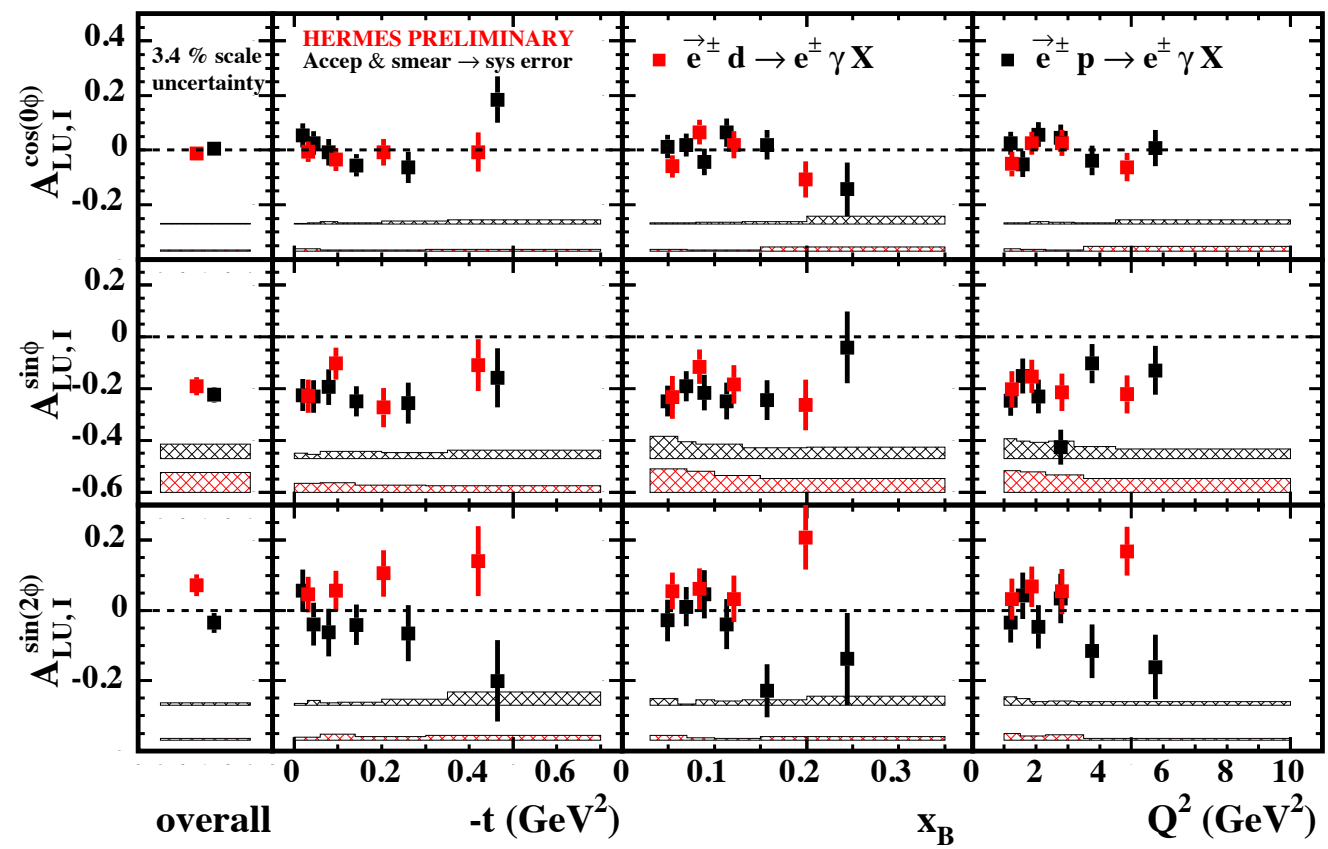

FIGURE 2. Comparison of the extracted BSA amplitudes from HERMES unpolarised proton and deuteron data as a function of $-t, x_{B}$ and $Q^{2}$. 